\title{
Parallel Centerline Extraction on the GPU
}

\author{
Baoquan Liu ${ }^{1}$, Alexandru C. Telea ${ }^{2}$, Jos B.T.M. Roerdink², Gordon J. Clapworthy ${ }^{1}$, \\ David Williams ${ }^{2}$, Po Yang ${ }^{1}$, Feng Dong ${ }^{1}$, Valeriu Codreanu ${ }^{2,3}$ and Alessandro Chiarini ${ }^{4}$ \\ ${ }^{1}$ University of Bedfordshire $\quad{ }^{2}$ University of Groningen $\quad{ }^{3}$ Eindhoven University of Technology $\quad{ }^{4}$ SCS srl
}

\begin{abstract}
-
Centerline extraction is important in a variety of visualization applications including shape analysis, geometry processing, and virtual endoscopy. Centerlines allow accurate measurements of length along winding tubular structures, assist automatic virtual navigation, and provide a path-planning system to control the movement and orientation of a virtual camera.

However, efficiently computing centerlines with the desired accuracy has been a major challenge. Existing centerline methods are either not fast enough or not accurate enough for interactive application to complex 3D shapes. Some methods based on distance mapping are accurate, but these are sequential algorithms which have limited performance when running on the CPU. To our knowledge, there is no accurate parallel centerline algorithm that can take advantage of modern many-core parallel computing resources, such as GPUs, to perform automatic centerline extraction from large data volumes at interactive speed and with high accuracy.

In this paper, we present a new parallel centerline extraction algorithm suitable for implementation on a GPU to produce highly accurate, 26-connected, one-voxel-thick centerlines at interactive speed. The resulting centerlines are as accurate as those produced by a state-of-the-art sequential CPU method [40], while being computed hundreds of times faster. Applications to fly-through path planning and virtual endoscopy are discussed. Experimental results demonstrating centeredness, robustness and efficiency are presented.
\end{abstract}

Index Terms-Centerline, parallel algorithm, GPU techniques, virtual endoscopy.

\section{INTRODUCTION}

Centerlines are powerful descriptors of 3D shapes that exhibit local circular symmetry [12]. Biomedical shapes that are effectively described by centerlines include blood vessels, intestines, and airways. In this context, centerlines are useful for virtual navigation, such as virtual endoscopy using the colon centerline to control the movement and orientation of the virtual camera. Accurate length measurements and navigation through other tubular organs, such as the aorta or coronary arteries [30], also require centerline computations. The problem of finding the optimal paths of minimal collision probability for virtual engineering and architectural applications also involves centerlines [17].

Although the concept of a centerline is quite intuitive, its mathematical definition is not unique. The concept of a centerline (also known as a medial or symmetry axis) was first introduced by Blum [10]. Based on previous work [3, 7, 8, 12,39,40], an adequate centerline definition should meet at least the following three requirements: Connectivity, Centeredness and Singularity. Connectivity requires that the centerline of a compact shape is also a compact object. For sampled objects, such as voxel models, this implies that centerlines of compact objects are sets of connected voxels. Centeredness requires that the centerline should be locally centered within the input shape with respect to the object boundary. Singularity requires that the centerline should be a single 1D path with no branches or selfintersections, and as thin as the sampling representation permits. For volumetric data, centerlines should be one voxel thick. A further requirement that there be no self intersections is desirable for some applications, such as path planning in virtual endoscopy.

Centerlines are closely related, but not identical to, other shape descriptors such as surface and curve skeletons. Unlike a centerline, the surface skeleton $\mathscr{S}$ of a $3 \mathrm{D}$ shape $\Omega \subset \mathbb{R}^{3}$ is uniquely defined as the locus of the centers of maximally-inscribed 3D spheres in $\Omega[3,27]$. This results in $3 \mathrm{D}$ surfaces or $2 \mathrm{D}$ manifolds. In contrast to surface

Manuscript received 31 March 2007; accepted 1 August 2007; posted online 27 October 2007.

For information on obtaining reprints of this article, please send e-mail to: tvcg@computer.org. skeletons, curve skeletons $\mathscr{C}$ are loosely defined as 1D structures with branches, which are locally centered within, and homotopic with, the input shape $\Omega$. The lack of a universally accepted formal definition has led to a situation in which the various methods that compute curve skeletons use different definitions and thus produce differing results $[3,4,27,30,37]$.

Centerlines share features such as centeredness, connectivity and 1D dimensionality with curve skeletons. However, unlike a curve skeleton, a centerline $\mathscr{L}$ is typically defined as a single path with no branches or self-intersections. An additional requirement is that a centerline passes through two user-selected end points $\mathbf{S} \in \partial \Omega, \mathbf{E} \in \partial \Omega$ on the surface $\partial \Omega$ of the object $\Omega[7,8,39,40]$. This allows users to confine the extracted centerline to only a desired part of the shape $\Omega, e . g$., for a controlled measurement of a branchless 1D fly-through path. An example centerline is shown in Fig. 1; while the two end points can be arbitrarily chosen on the object surface by the user (or be detected automatically, too), the resulting centerline between them quickly tends towards the local center of the object.

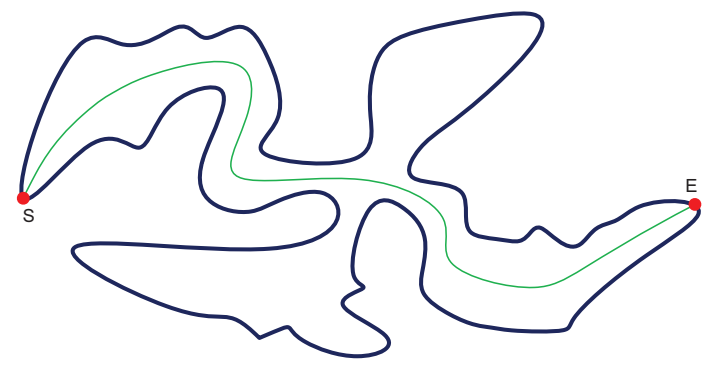

Fig. 1. An example centerline connecting two user-defined endpoints (source and end points), which allow a particular segment of the object to be specified for fly-through path planning.

In summary, the difference between a centerline $\mathscr{L}$ and a curve skeleton $\mathscr{C}$ lies in two aspects: (1) a centerline is a single path with no branches, while a curve skeleton is a $1 \mathrm{D}$ topological structure with branches; (2) a centerline must pass through two arbitrary given end points on the object surface $\partial \Omega$, so its form is dependent upon the selection of these points, whereas a curve skeleton is uniquely deter- 
mined simply by the object's shape.

Hence, while a curve skeleton is fully located inside the object (except for some special cases, such as 1- or 2-voxel wide parts of objects which cannot be traversed by a voxel-thick curve without touching the surface $\partial \Omega$ ), a centerline must start from, and end on, the surface $\partial \Omega$. As a compact representation of an object's topological structure, a curve skeleton is well suited to $3 \mathrm{D}$ animation, morphing, matching and registration [13]. In contrast, centerlines are more useful in visualization applications such as fly-through path planning between user-specified end points.

Ideally, a centerline extraction algorithm should generate a good approximation to the central path through a shape $\Omega$, i.e., the centerline $\mathscr{L}$ should be as close as possible to the corresponding parts of the curve skeleton $\mathscr{C}$ of $\Omega$, apart from in the regions close to where $\mathscr{L}$ connects to its endpoints on $\partial \Omega$. Centerlines should be computable at interactive rates even for large and complex objects sampled at high resolutions. This last constraint is especially important when considering the potential deployment of centerlines in a clinical environment, where interactive performance is highly desirable. For instance, a recent detailed evaluation of the quality of centerlines used for coronary artery exploration considered 14 centerline extraction methods deemed suitable, from an accuracy perspective, for medical applications [30]. Typical running times of these methods range between tens of seconds and hours per dataset, which clearly makes them unsuitable for interactive exploration.

In virtual colonoscopy, centerline extraction provides a compact colon shape description and enables accurate colon geometry measurement for both pre-planned and interactive navigation. During treatment, problems involved in issues such as precise polyp registration between virtual and "real" fiber-optic colonoscopy or shape registration between supine and prone $\mathrm{CT}$ scans can be overcome by using centerlines. However, extracting centerlines from large voxel volumes while satisfying centeredness, singularity, and computational efficiency remains very challenging.

Many methods have been proposed for the computation of centerlines, either from 3D (CT or MRI) [30] scans or from 3D polygonal models [36]. The most efficient and accurate centerline extraction method is considered to be that based on Euclidean distance mapping $[7,8,39,40]$. Here, centerline points are forced to lie as far from the object boundary as possible by the use of a distance-fromboundary (DFB) field. This ensures that the centerline is a subset of the surface skeleton $\mathscr{S}$, which is located along the DFB maxima [27]. However, this method is a canonical serial algorithm with fundamental dependency between iterations. It does not readily lend itself to parallelization as it relies on a sorted priority queue and can evaluate only one voxel (i.e., the head of a queue) for each iteration. As a result, the algorithm cannot take advantage of the multiple parallel computing cores now available on the CPU or GPU, as discussed further in Section 3.

The main contribution of this paper is a new parallel centerline extraction algorithm suited to modern GPU hardware. Our algorithm adopts the general DFB-based centerline extraction approach [40], which is regarded as state of the art. However, instead of evaluating the nodes serially, we propose that multiple node-evaluations are performed concurrently on parallel computing cores by using a parallel wavefront propagation technique. This allows the new algorithm to be implemented in a parallel GPGPU environment. Our experiments have shown that the proposed GPU algorithm produces an accurate centerline with the same quality as the original algorithm, but it runs hundreds of times faster, thus allowing centerline extraction to be performed at interactive rates. This level of interactivity makes a fundamental difference to the way in which applications such as virtual path planning can be performed.

\section{Related Work}

The problem of centerline extraction has received a great deal of attention over many years, and a vast number of centerline calculation algorithms have been described in the literature.

The early manual marking method [15] requires intensive manual intervention as the user has to indicate key points at the center of each object region on each of the several hundred axis-aligned 2D slices of a volume dataset. The algorithm then computes the centerline curve by interpolation. This method is simple, but time-consuming and tedious in operation, and its results are by no means more accurate than those produced by automatic methods. Because the colon is usually visualized on a 2D screen, it is difficult for users to locate points that are centered inside it, so this method is highly liable to human error.

Topological thinning methods remove voxels on the boundary $\partial \Omega$ while preserving connectivity and topology $[6,24]$. Voxel removal in distance-to-boundary order enforces centeredness $[3,26]$. The resulting structure, typically either the surface skeleton $\mathscr{S}$ or curve skeleton $\mathscr{C}$ of $\Omega$, can next be simplified, or pruned, in order to extract the desired centerline $\mathscr{L}$ [28]. Although the thinning procedure is conceptually simple, its requirement for a distance-driven ordering, topological checking and postprocessing, can make the entire procedure quite intricate and time-consuming [3]. A 3D thinning implementation for ITK by Homann [19] is quite popular in the medical imaging community.

Voronoi diagram methods [1] find centerlines as the paths in Voronoi diagrams that minimize the integral of the radii of the maximal inscribed spheres along the path; this is equivalent to finding the shortest paths in the radius metric. To perform the calculation, a wave is propagated from a source point (one centerline end point) using the inverse of the radius as the wave speed, and the wave arrival time is recorded at all points of the Voronoi diagram; the line is then backtraced from a target point (the other end point of the centerline) along the gradient of the arrival times. The propagation can be described using the Eikonal equation and is computed using the fast marching method [32]. This method was implemented in the Vascular Modeling Toolkit (VMTK) [2], but this has only a CPU version. This method is accurate but too slow for interactive application. Indeed, computing an accurate Voronoi diagram of the input shape is equivalent to computing its surface skeleton $\mathscr{S}$, and this computation is not interactive, even in recent GPU implementations [20,22].

Distance mapping approaches were first used in robotic path planning [21]. They are generally considered to provide the fastest solution among the different categories. Such methods have two phases. The first phase computes the distance from a user-specified source point $\mathbf{S}$ to each voxel inside the 3D object, called the DFS (distance from source) map. In the second phase, the shortest path from the other end point $\mathbf{E}$ to the source point is found by descending through the gradient of the DFS map. The shortest path can be rapidly extracted by a simple backtracing. Conceptually and algorithmically, this approach is essentially the same as the method for computing geodesics between two given points on a surface [25].

Previous centerline algorithms using distance mapping differ in how they specify the distances between orthogonal, 2D-diagonal, and 3D-diagonal neighboring voxels. The most accurate distance measure is the Euclidean metric, but most algorithms use approximate distance transformation metrics such as the Manhattan metric $[7,8,39,40]$ in order to reduce the computational cost. Distance mapping approaches often employ Dijkstra's shortest path algorithm on the voxel connectivity graph to extract the centerline fully automatically.

Although distance mapping can meet the connectivity and singularity criteria well, in many implementations its centeredness is often less than perfect as the resulting path tends to 'hug' the concave corners of $\partial \Omega$ at sharp turns (i.e., the tendency of the centerline $\mathscr{L}$ to deviate from the curve skeleton $\mathscr{C}$ and to approach $\partial \Omega$ in areas where $\mathscr{C}$ has sharp turns) [40]. This is not surprising when we consider the above-mentioned analogy between centerlines and geodesics of the DFS field. Efforts have been made to push the shortest path towards the object center by post-processing [41], but this does not completely solve the problem. Similar issues arise when computing curve skeletons using distance-from-boundary (DFB) fields [4]. Bitter et al. [7,8] proposed a penalized-distance algorithm to extract centerlines by adding a penalty value to the distance cost at each node to keep the centerline away from the boundary. The algorithm proposed by Wang et al. [40] solves the "corner-hugging" problem simply 
and effectively by employing the DFB field as node-weights and extracting the centerline from a minimum-cost spanning tree (MST) built from the DFB field. This efficient and robust algorithm is fully automatic and can generate accurate centerlines, but it has three properties inhibiting parallelization: a fundamental loop dependency, limited explicit parallelism, and excessive synchronization. We discuss these issues further in Section 3.

Level set methods $[16,38]$ have been found to produce accurate centerlines. However, they are not fast enough for interactive applications.

Smistad et al. [35] introduced an efficient GPU-based airway tree segmentation algorithm that extracts centerlines by performing a ridge traversal on the result of a tube detection filter. The ridge traversal approach, first proposed by Aylward et al. [5], is performed on filtered images, so it can have accuracy problems due to its sensitivity to noise, local artifacts and its initialization, as mentioned in [33,35]. Also, this algorithm remains completely serial in form, so it is difficult to increase its speed by GPU parallelization.

Recently Smistad et al. [34] introduced a new model-based Tube Detection Filter (TDF) combined with a new parallel centerline algorithm which can extract centerlines directly from the TDF result. Their results show that the method is able to extract airways and vessels in 35 seconds on a high-end GPU, and their parallel centerline extraction algorithm is 1-2 seconds faster than the serial ridge traversal extraction algorithm.

Several voxel-based methods have been proposed to compute Euclidean surface and/or curve skeletons of 3D data [3, 4, 14, 18, 27]. A recent qualitative comparison of curve skeleton techniques is given in [36]. However, these methods do not produce single-path centerlines between user-specified endpoints. It is conceivable that such methods could be post-processed to extract centerlines, for example by finding the shortest-path linking the specified endpoints which also passes through an extracted curve skeleton. However, none of these methods is fast enough for near-real-time curve skeletonization of large 3D volumes, and the postprocessing could be tedious and timeconsuming, so they fail our interactive requirement.

Bleiweiss [9] introduced a GPU implementation of parallel pathfinding for many thousands of agents in crowded game scenes in which each individual agent runs a separate pathfinding algorithm (to find its own individual path) in a single GPU thread. Therefore, the parallelism lies in the multiple agents' multiple pathfindings, which is a coarse granularity parallelism. In contrast, our parallel algorithm exhibits fine granularity parallelism, with multiple GPU threads working together to find a single path for a complex-shape object.

\section{REVIEW OF THE SERIAL ALGORITHM}

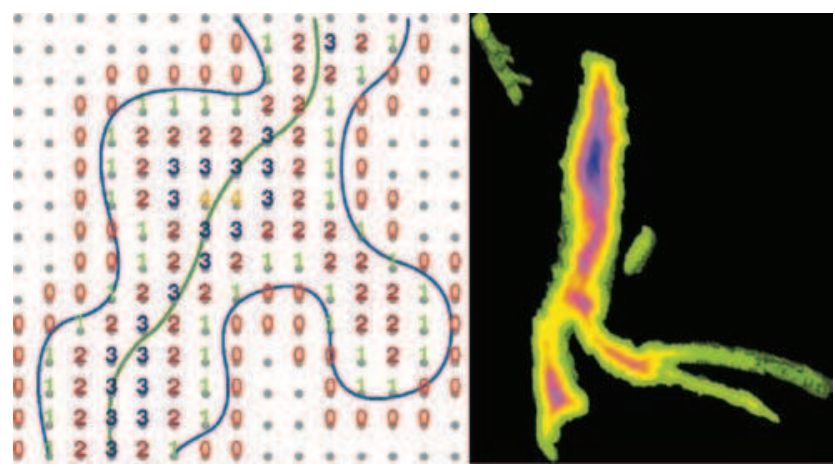

Fig. 2. Examples of DFB shown in 2D slices. Left: explicit DFB values; right: an aorta DFB field visualized through a color map (image courtesy of Bitter et al. [7]).

In this section, we first review the serial algorithm introduced by [40]; this will be referred to hereafter as serial). Our new parallel algorithm is then introduced incrementally in the following sections.
In the serial algorithm, the centerline is defined as the minimumcost path spanning the 3D DFB field inside the input object. An example DFB field is shown in Fig 2. This is a concise and concrete definition, similar to the traditional centerline description given by Blum [10], but it is more practical for implementation. The serial algorithm can produce very accurate centerlines based on the DFB field, in which the Euclidean distance between each interior voxel and the object boundary is recorded.

The serial algorithm consists of two main steps. First, it builds a minimum-cost spanning tree (MST) in the DFB field, then it finds the sequence of voxels that lie along the centerline by backtracing from the end point to the start point through the previously constructed MST. The first step is by far the costlier in terms of computation. Centeredness is assured by the fact that the DFB field uses exact Euclidean distances, computed by a fast algorithm proposed by Saito et al. [29] and described also by Meijster et al. in [23].

The serial algorithm solves the centerline problem on graphs with nonnegative edge weights by looping over the contents of a priority queue until all nodes have been evaluated. The regular sampling grid of the DFB field is treated as a 3D directed weighted graph. Each voxel corresponds to a graph node. Graph edges are given by the 26-neighbor relations between adjacent voxels. Each edge has two directions pointing towards its two respective end points, and each direction has its own weight, called the DFB-cost, equal to the inverse of the DFB of the end voxel to which it points.

The MST is defined as a tree that connects all of the interior voxels (that is, those in $\Omega \backslash \partial \Omega$ ) at the minimum DFB-cost. To build this MST efficiently, a modified Dijkstra technique is used. Given a source point $S$ and an end point $E$, both specified interactively by the user on the object surface $\partial \Omega$, the MST is built by the following four-step process:

1. Mark $S$ as visited and define it as the current node $C$. Set the pathlink of $S$ to NULL, where pathlink is used to label a node's predecessor in a path.

2. For the current node $C$, insert the unmarked 26-neighbors $B$ of $C$ into a priority queue $Q$ sorted on the DFB-cost, and set their pathlinks to $C$.

3. Remove the head node from $Q$ (that is, the one with the minimum DFB-cost), mark it as visited, and define it as the current node $C$.

4. Repeat from step 2 until $Q$ is empty. After this, all nodes have been evaluated.

The algorithm constructs, for each interior voxel $\mathbf{p} \in \Omega \backslash \partial \Omega$, a pathlink pointing toward its neighboring voxels, through which $\mathbf{p}$ reaches the source point $S$ with a minimum DFB-cost. At each iteration, the priority queue must be kept sorted (either by explicit re-sorting or by ensuring that any insertion retains the overall ordering) in order to find the node $C$ that has the minimum DFB-cost. This node is used to launch the next iteration, which will, in turn, insert new nodes (i.e., $C$ 's neighbors) into the queue.

The above algorithm is completely serial, and its speed cannot be increased by the use of parallel hardware due to the fundamental loop dependency caused by the use of a priority queue. Specifically, to ensure a correct MST construction, based on the greedy rule that information is propagated only from the node with the minimum DFB-cost to other nodes at any time, only a single node can be processed at each iteration to update its 26 neighbors. Furthermore, processing the node $C$ at the head of the queue causes its neighbors to be inserted into the queue which, in turn, requires the queue to be re-ordered to ensure that the minimum DST-cost node is at the front.

This process exhibits excessive synchronization: at every iteration only the head node can be processed, and each node-evaluation has to wait for the completion of the sorting operations from the last iteration, so multiple node evaluations cannot be performed concurrently. Apart from the above, a further bottleneck is presented by the frequent sorting of the queue. Each sorting operation is performed at best in $O(\log M)$, where $M$ is the average number of elements in the priority 
queue at each iteration. Moreover, the total number of iterations we need to loop in this algorithm is equal to $K=\|\Omega\|$, the number of the foreground voxels inside or on the 3D shape $\Omega$ (rather than $N$, i.e., the total number of voxels in the volume), since all $K$ voxels need to be removed from the queue (one at each iteration) in order to propagate information throughout the object. So the complexity of the whole algorithm is $O(K \times \log M)$.

\section{QUEUE-FREE SERIAL ALGORITHM RUNNING ON THE CPU}

As a first step towards parallelization, we introduce in this section a queue-free serial algorithm running on the CPU based on the same DFB field as the original serial algorithm in Sec. 3. In the following sections, we show how we parallelize this queue-free algorithm for the GPU by allowing parallel evaluations of multiple nodes.

As explained in Sec. 3, the original serial algorithm [40] is not parallelizable due to its fundamental loop dependency, which is caused by the use of a priority queue. To bypass this problem, here we first introduce a new brute-force serial algorithm (referred to as $b f_{-} s$ ) which does not use a priority queue. This new queue-free algorithm is based on the same accurate Euclidean DFB field as serial and again assumes that the object interior is a 26-connected region. The DFB field is stored as a signed floating-point distance field, that is, it has positive values for voxels inside $\partial \Omega$, zero values on $\partial \Omega$, and negative values outside $\partial \Omega$.

The DFB-cost field is stored as a $3 \mathrm{D}$ read-only volume $D F B_{\text {cost }}$. A second 3D floating-point buffer, weight, is used to store, for each voxel, the accumulated weight value along its path originating from $S$. Each element of weight is initialized to a very large constant, MaxFloat, except the source point $S$, whose weight $[S]$ is set to 0 . As the algorithm proceeds, we compute the accumulated weight from a source point $S$ to each of its neighbors $B$ using the formula weight $[B]=$ weight $[S]+D F B_{\text {cost }}[B]$. The neighbor nodes then propagate their weight values progressively to their own neighbors until $E$ is reached.

To construct a correct MST, our new algorithm ensures that the weight values are propagated only from nodes with smaller weights to nodes with larger weights. Thus, the weight values of individual nodes can only decrease monotonically as a result of the node evaluations during the iterations, all node weights (except $S$ ) will be updated from MaxFloat to smaller values in a monotonically decreasing manner. In this way, the MST can be constructed in a "downwind" direction proceeding from the source point $S$ to the end point $E$.

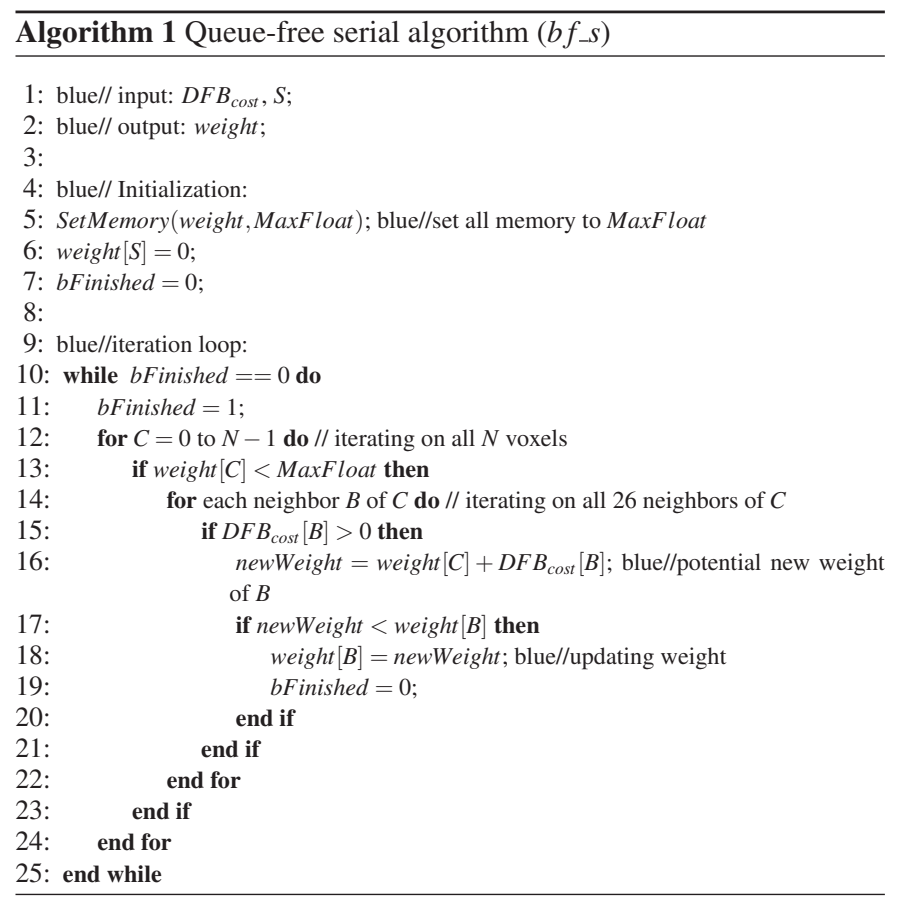

The pseudo-code of $b f_{-} s$ is shown in Algorithm 1. In detail, we proceed as follows:

1. At initialization, the source point $S$ is selected by the user, and weight $[S]$ is set to zero. A Boolean value (bFinished), used to indicate termination, is also set to 0 .

2. At each iteration, if bFinished $==0$, we reset it to 1 , and then launch a for-loop to iterate on all $N$ voxels of the input volume. Here, we propagate the weight information from each visited voxel $C$ to its 26-neighbors. Each neighbor $B$ of $C$ has its weight $[B]$ value updated to weight $[C]+D F B_{\text {cost }}[B]$ if this value is smaller than weight $[B]$, which ensures that weight $[B]$ can only decrease. If any weight update is successful, we set bFinished to 0 , which means we need next to propagate information from $B$ to its own neighbors at the next iteration.

3. Step 2 is repeated until no further weight updates occur (bFinished =1). At this point, the final MST is available in the weight dataset.

The number of iterations is bounded because, after the propagation has reached all of the interior nodes and no weights remain to be updated, the iteration procedure will terminate.

At each iteration, this algorithm evaluates $N$ voxels, where $N$ is the total number of voxels in the input 3D volume, whereas serial evaluates only the nodes that were removed from its priority queue. The number of these nodes $K=\|\Omega\|$, that is, the voxels on or inside the 3D shape (i.e., the foreground voxels). As a result, our brute-force algorithm $b f_{-} s$ is slower than serial, since $K<N$, so $b f_{-} s$ is uncompetitive on a serial processor.

However, $b f_{s} s$ is queue free and has no loop dependency. As such, it is completely parallelizable, leading to the possibility of a highlyefficient GPU-based centerline extraction method, as discussed in the next section.

We also note that the centerlines produced by the serial and $b f_{-} s$ algorithms may be slightly different as the respective MSTs are created in slightly different ways. In $b f_{-} s$, the weight values are not explicitly sorted, but are accumulated (weight $[C]+D F B_{\text {cost }}[B]$ ) and updated in a monotonically decreasing manner. The final path connecting the source to destination nodes is found by tracing paths in the gradient of the accumulated weight field. In contrast, serial sorts the weights explicitly (but does not accumulate them), and uses a pathlink to connect the sorted nodes after the weight-ranking to construct the MST. The differences in the MSTs are very small and lead to insignificant centerline differences, as we show in Sec. 6.

\section{GPU PARALLELIZATION}

We next present two parallel implementations of the $b f_{-} s$ algorithm using the GPU. The key idea behind both implementations is to allow multiple node evaluations to be performed concurrently using the many computing cores available on a GPU. Both implementations store the $D F B_{\text {cost }}$ field as a 3D GPU texture. After the MST is constructed in parallel on the GPU, we transfer it to the CPU, where we construct the final centerline by simply backtracking in the MST from the endpoint $E$ to the starting point $S$.

The first implementation ( $b f_{-} p$, Sec. 5.1) is a brute-force parallelization, which is simple to implement, while the second implementation ( im $_{-}$, Sec. 5.2) is more involved, but considerably more efficient. Both produce results identical to those of $b f_{-} s$.

\subsection{Brute-force parallel algorithm}

The brute-force parallelization $b f_{-} p$ is a direct mapping of the queuefree serial algorithm $b f_{-} s$ onto the GPU, where we evaluate all $N$ voxels of the input volume in parallel at each iteration. The pseudo-code of $b f_{-} p$ is shown in Algorithm 2. In detail, we proceed as follows:

1. The initialization is identical to that of $b f_{s} s$. 
2. At the beginning of each iteration, if $b$ Finished $==0$, we reset it to 1 , and then launch a CUDA kernel in exactly $N$ GPU threads. The thread associated with node $C$ propagates the weight information from $C$ to its 26-neighbors. To ensure that the weight of any neighbor $B$ can only decrease, we use CUDA's atomic function atomicMin(). If an update of $B$ is successful, we set bFinished to 0 . The iterations are repeated until bFinished $=1$ at the end of an iteration.

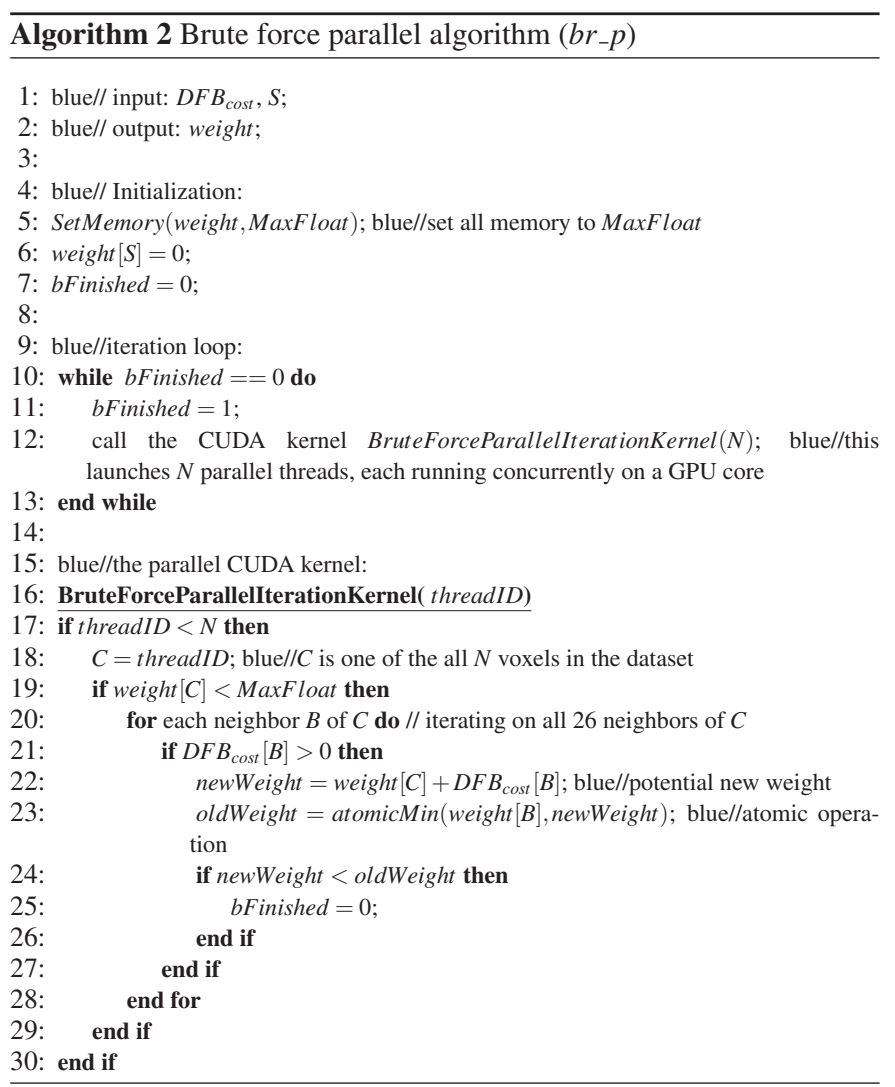

The CUDA atomic operation atomicMin() is used to update the weight buffer in a parallel manner (Alg. 2, line 20) so as to avoid a race condition, which would occur when multiple threads attempt to update the same buffer at the same time.

The most obvious difference from $b f_{-} s$ is that, at each iteration, all the nodes propagate information simultaneously to their neighbors in $b f_{-} p$, rather than processing taking place a single node at a time. The drawback of $b f_{-} p$ is that we have to launch many CUDA threads $-N$ threads to evaluate all $N$ voxels at each iteration.

However, a high proportion of these node evaluations are unnecessary because many voxels are either inactive (i.e., weight was not changed at the last iteration) or are exterior to the object $\Omega$ (i.e., their $D F B_{\text {cost }}<0$ ). Processing such voxels will not contribute to the information propagation; further, threads will have substantially different execution times, which is an inefficient use of the resources.

Nevertheless, this scheme does have the virtue of keeping the mapping of threads to voxels simple.

\subsection{Improved parallel algorithm}

In contrast to the brute-force parallel algorithm $b f_{-} p$, which evaluates all $N$ voxels by launching $N$ GPU threads at each iteration, the improved parallel algorithm $i m_{-} p$ presented in this section processes only the active voxels that must be evaluated, that is, only those with the potential to update their neighbors. Inactive nodes are skipped, greatly reducing the number of threads that have to be launched.

In $i m_{-} p$, we again perform multiple node-evaluations concurrently for each iteration, but we now use a wavefront propagation scheme,

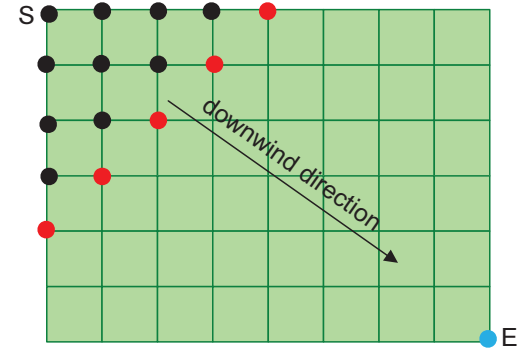

Fig. 3. Downwind wavefront propagation from source point $S$ to end point $E$ in parallel mode, so that at each iteration only the currently active nodes (red) propagate their weights to their neighbors simultaneously.

as shown in Fig 3. The algorithm starts from the source point $S$, and at each iteration, a 1D array currentArray records the ID of the active nodes. These are the nodes whose weights were changed at the last iteration and which will update their neighbors' weights at the current iteration. Conceptually, this scheme is similar to the narrowband structure used by the fast marching method [32].

At each iteration, we use a ping-pong technique, with two 1D arrays, currentArray and nextArray, being interleaved for consecutive iterations. currentArray stores the active nodes generated from the previous iteration and is used to launch the current iteration. nextArray stores the new active nodes generated at the current iteration and is used to launch the next iteration. Hence, whenever a node's weight is changed, it will be recorded in nextArray at the current iteration, and will provoke the launch of a thread at the next iteration. To avoid a node being added to the same array multiple times during the parallel processing, a Boolean 3D array (mark) is used to record if a node has already been added to that array. All elements of mark are set to zero at the beginning of each iteration.

Active nodes behave like a wavefront, with information being propagated from the source node $S$ to all other nodes in parallel. We classify the grid points into three groups: discovered points, which were previously active but are not currently so (shown in black in Fig. 3); active points, whose weights were updated in the previous iteration (shown in red); and undiscovered points, which have not yet been visited. At each iteration, we launch threads only for active points - one thread for each active point. For a volume of a few million voxels, the number of active points (and thus also of the threads launched) is typically in the order of thousands, which is much lower than the millions of threads required by $b f_{-} p$.

The pseudocode of $i m_{-} p$ is shown in Algorithm 3. In detail, we proceed as follows:

1. Given a source point $S$, we set weight $[S]$ to zero, append $S$ to currentArray, and set the array's length currentLength to 1 . This array records all currently active nodes; these are the nodes that will propagate their weight information to their neighbors at the current iteration.

2. At each iteration, if currentLength $>0$, we launch the parallel CUDA kernel on exactly currentLength GPU threads, one thread per element $C$ of currentArray. Each thread propagates the weight information from $C$ to its 26-neighbors, as in the brute force algorithm $b f_{-} p$. If an update of a neighbor $B$ is successful, we test if $B$ is already marked. If not, we mark it, append it to nextArray, and increment its length, nextLength. Using mark avoids adding $B$ to nextArray more than once.

3. We swap currentArray and nextArray, and clear mark and nextLength, so that we can perform the next iteration. Steps 2 and 3 are repeated until currentLength $=0$, i.e., until all nodes have been discovered and no node can be further updated.

Our improved $i m_{-} p$ algorithm performs identically to the $b f_{-} p$ algorithm, apart from skipping all of the unnecessary node evaluations, 


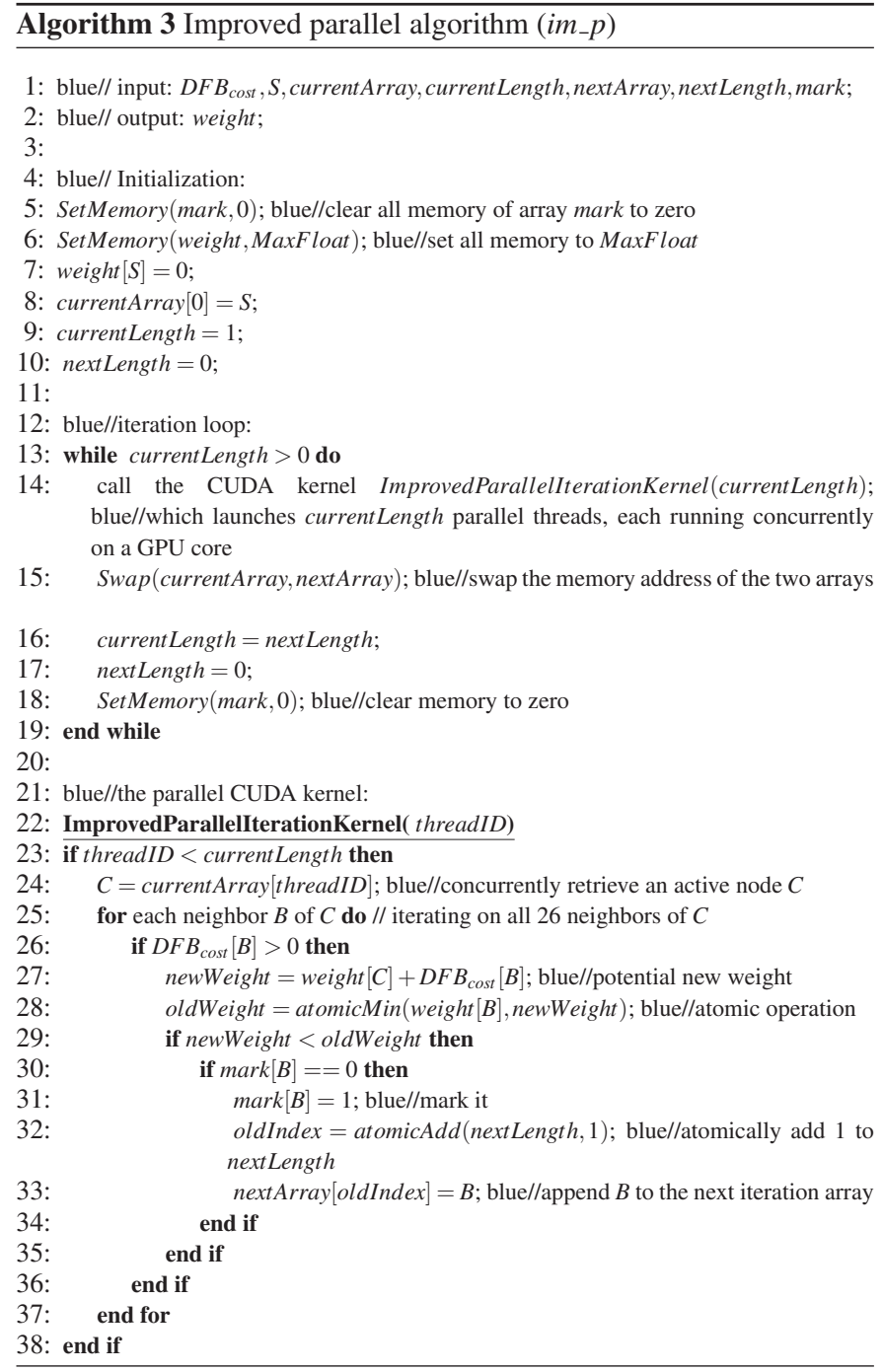

so it produces exactly the same results as the $b f_{-} s$ and $b f_{-} p$ algorithms. We have confirmed this in practice by computing voxel-wise comparisons of the results of the three algorithms.

The added value of $i m_{-} p$ over the previous algorithms lies in three factors: (a) we do not need to maintain an expensive sorted queue; (b) instead of evaluating nodes one at a time, we evaluate multiple nodes in parallel on the GPU - a deterministic result is ensured by use of monotonically decreasing information propagation and by CUDA's atomic operations; (c) at each iteration, only currently active nodes are evaluated, as opposed to the entire voxel set as in the brute force algorithms.

\subsection{Centerline extraction by backtracing the MST}

After the iterations in Algorithm 2 or 3 have terminated, the MST is available in the weight buffer. Having performed the computeintensive calculations on the GPU, the remainder of the algorithm is performed on the CPU. We thus transfer weight from the GPU memory to the CPU memory and extract the centerline on the CPU by a simple backtracing which traverses the MST from the target end point $E$ along the gradient direction of the weight field until the source point $S$ is found.

In detail, at each step $i$ of the traversal, we test the 26-neighbors of the current node $C_{i}$ (initialized as $C_{0}=E$ ), and choose the one with the minimum weight as the next current node $C_{i+1}$. We repeat this process until we find the source point $S$ as the last node $C_{n-1}$. The desired centerline is formed by the point sequence $\left[C_{0}, C_{1}, C_{2}, C_{3}, \ldots, C_{n-1}\right]$. The number of steps of this process, equal to the length of the centerline, is of $O(D)$ where $D$ is the diameter of the object, which is typically of the same order as the size of the space, i.e., $\sqrt[3]{N}$ for a volume of $N$ voxels [27]. This step is very fast, and can be executed in real-time on the CPU.

Our approach could also be used to extract general 3D curve skeletons including all branches. Since the MST connecting all of the interior voxels has already been built, it is straightforward to find all other possible branches from the MST using the branch-finding method introduced in [40], and then merge them all into one topology.

\section{Results}

The parallel algorithms presented above were implemented using CUDA 5 and tested on a PC computer with an Intel $3.5 \mathrm{GHz}$ CPU and an NVIDIA GeForce GTX 690 graphics card. Although the GTX 690 has two GPUs, one is sufficient to handle all of our datasets, resulting in a usage of 2 GB video memory and 1536 CUDA cores. For examination and comparison, the centerlines extracted were displayed at a screen resolution of $1024 \times 768$ pixels.

As in previous serial approaches [40], our method can extract centerlines from either volume data (CT or MRI) or polygonal mesh models, as long as a 3D DFB field (as input to our centerline algorithm) is pre-computed from the original object data, either from the segmented medical image slices or from the polygonal mesh.

The DFB pre-computation can be performed efficiently by recent GPU-based Euclidean distance transforms [11,31] - for example, within a second for a volume of $512^{3}$ voxels on the hardware indicated above. The DFB pre-computation needs to be performed only once for a given model, after which the user can interactively specify the desired centerline start and end points and extract the actual centerlines. Since the focus of this paper is on centerline computation, we exclude the DFB pre-computation time from our runtime performance comparisons.

In our experiments, the user is presented with the input 3D surface, rendered semi-transparently. The user can next define the two end points by interactively clicking any two points in this rendered image. The selected points are shown as blue squares in all of the resulting images and videos. For each of the user's clicks, the program will pick the 3D point on the frontmost surface layer of the object under the selected pixel, which is then used as $S$ or $E$.

After $S$ and $E$ are defined, the centerline extraction algorithm is automatically applied, and the resulting centerline points are displayed in real-time. Note that the two points selected must belong to a connected component of the object, otherwise an MST cannot be built connecting them. The interactive features of the algorithm can be seen in the accompanying videos. For comparison, the resulting images show the centerlines produced by serial, $b f_{-} p$, and $i m_{-} p$ rendered in black, blue and red, respectively.

No parameters need to be set by the user at run-time, apart from the specification of the start and end points. For the $b f_{-} p$ algorithm, threads are scheduled as 3D thread blocks on the GPU, with each block having $4 \times 4 \times 4$ threads. For the im_ $p$ algorithm, threads are scheduled as 1D thread blocks, with each block having 16 threads. We found that these are the optimized configurations and subsequently used them as presets in all our experiments.

\subsection{Quality comparison}

For all the shapes tested, the new algorithms managed to extract a visually correct and plausible centerline. In particular, we found that the centerlines respected the centeredness criterion well - no collision of the centerline with the input surface $\partial \Omega$ was found when directly examining the centerlines or using them as fly-through paths.

Since there is no such thing as the "best" centerline [18], the question of which centerline one prefers depends upon the criteria that are imposed by the desired application. For some datasets, the results of the serial and newly proposed methods are completely identical (see Figures 4 and 5). For other datasets, there are small differences between the serial and our new queue-free methods, as shown in Figure 6 . 
The key point is that the three queue-free methods $\left(b f_{-} s, b f_{-} p, i m_{-} p\right)$ are guaranteed to produce exactly the same results, and these results at worst show only very minor differences from those produced by the original queue-based serial method. This proves that both parallel algorithms are robust, and their results are steady and deterministic. Because of this, the results of $b f_{-} s$ and $b f_{-} p$ are not shown in Figure 6 to avoid redundancy.

Figure 4 shows results for the blood vessel model at three different resolutions. It also shows that the higher the data resolution is, the smoother the centerline will be, though the computation time will be longer (see Table 1). In Figure 5, the three algorithms were tested on a cuboid and a doll model. Figures 4 and 5 demonstrate that the three algorithms produce identical centerlines for these simple-shaped models. These experiments were run multiple times, and all three algorithms always produced the same centerlines, as tested by a voxelby-voxel comparison.

For other complex models, the voxel-by-voxel comparison indicated that there can be some differences between the centerlines produced by the serial and parallel algorithms. However, these differences are so small that they are not easy to notice, as can be seen in the examples in Figure 6.

The reason behind the differences is that the weight values are accumulated in our parallel algorithms, but not in the serial algorithm, as explained in Section 4. The serial algorithm uses a pathlink for each voxel to label its predecessor. In contrast, the parallel algorithms use the gradient direction of the accumulated weight-field to find a voxel's predecessor node.

As a desirable by-product of the weight accumulation, we note that the results produced by the parallel algorithm are smoother for complex models than those produced by the serial algorithm. This is because accumulating the weights acts as a low-pass filter of the resulting centerline. This effect is especially obvious for the dinoSkeleton model in Figure 6, where the surface of the tail of the dinosaur exhibits high-frequency variations. These cause the serial algorithm to produce a centerline with high-frequency noise, whereas our parallel algorithm filters such noise and hence produces a smoother centerline. This shows that our method is less sensitive to small-scale perturbations of the input surface than the serial algorithm.

\subsection{Performance comparison}

We have performed extensive experiments on a wide range of datasets. The performance statistics corresponding to Figures 4, 5 and 6 are shown in Table 1. All algorithms were tested on the hardware mentioned at the beginning of Sec. 6. The serial code was our implementation based on the flowchart provided in [40].

From Table 1, it is clear that $i m_{-} p$ is up to 7 times faster than $b f_{-} p$, which is itself significantly faster than serial. On a broader front, if we compare our parallel centerline extraction $\left(i_{-} p\right)$ with recent mesh and volume-based curve-skeleton algorithms [3,20,27,35,37], we observe that $i m_{-} p$ is one to two orders of magnitude faster on similar hardware and for the same input datasets. As such, our algorithm presents concrete added value in an interactive context, as in such situations postprocessing the curve skeleton output of the latter algorithms would not be able to deliver interactive frame rates. Interestingly, our method is also faster than mesh-based curve-skeletonization algorithms [37], including carefully GPU-parallelized ones [20], which stand out for their high throughput.

\subsubsection{Performance analysis}

The improved parallel algorithm $i m_{-} p$ is much faster than any previous algorithm but it does not sacrifice accuracy for speed. Its high performance is due to the parallel execution of many concurrent CUDA threads on many GPU cores.

Our analysis showed that, as in all other MST-based centerline extraction algorithms, the bottleneck of our $i m_{-} p$ algorithm lies in the MST computation stage, which accounts for more than $90 \%$ of the total time. The MST backtracking is very light and accounts for less than $10 \%$ of the time.
Table 1. Performance comparison over a range of datasets. The table shows the data resolution, the length of the extracted centerline (number of nodes along the centerline), and the extraction time (in milliseconds) of the algorithms serial, $b f_{-} p$ and $i m_{-} p$, respectively. The respective centerlines extracted are shown in Figures 4, 5 and 6.

\begin{tabular}{|c|r|r|r|r|r|}
\hline dataset & $\begin{array}{r}\text { data } \\
\text { resolution }\end{array}$ & $\begin{array}{r}\text { centerline } \\
\text { length }\end{array}$ & $\begin{array}{r}\text { serial } \\
\text { (in ms) }\end{array}$ & $\begin{array}{r}\text { bf_p } \\
\text { (in ms) }\end{array}$ & $\begin{array}{r}\text { im_p } \\
\text { (in ms) }\end{array}$ \\
\hline vessel & $128^{3}$ & 124 & 297 & 47 & 31 \\
\hline vessel & $256^{3}$ & 245 & 13978 & 140 & 63 \\
\hline vessel & $512^{3}$ & 489 & 554506 & 1513 & 203 \\
\hline cuboid & $128^{3}$ & 125 & 119808 & 234 & 47 \\
\hline doll & $128^{3}$ & 116 & 21107 & 78 & 31 \\
\hline aneurism1 & $256^{3}$ & 326 & 1410702 & 1326 & 390 \\
\hline aneurism2 & $256^{3}$ & 396 & 530294 & 951 & 297 \\
\hline aneurism3 & $256^{3}$ & 284 & 227621 & 609 & 187 \\
\hline aorta & $512^{3}$ & 526 & 457707 & 1311 & 312 \\
\hline ben & $256^{3}$ & 268 & 241552 & 764 & 203 \\
\hline colon & $256^{3}$ & 848 & 1104908 & 1763 & 515 \\
\hline dino & $256^{3}$ & 277 & 56769 & 281 & 78 \\
\hline dinoSkeleton & $256^{3}$ & 252 & 125207 & 297 & 93 \\
\hline hand & $256^{3}$ & 209 & 1142770 & 561 & 249 \\
\hline horse & $256^{3}$ & 323 & 809395 & 952 & 234 \\
\hline knot & $128^{3}$ & 137 & 387085 & 359 & 140 \\
\hline octopus & $256^{3}$ & 163 & 122305 & 512 & 141 \\
\hline snake & $256^{3}$ & 665 & 181866 & 1139 & 250 \\
\hline toy & $256^{3}$ & 221 & 3477761 & 1092 & 343 \\
\hline
\end{tabular}

In terms of complexity, the serial algorithm requires $K$ iterations for an input shape with $K$ voxels, and at each iteration, it needs to sort $M$ elements in the priority queue. Hence, its complexity is $O(K \times \log M)$, as the sorting complexity is $O(\log M)$ at best. Here, $M$ is the number of active nodes currently in the queue, that is, $M$ is the length of the priority queue at each iteration. However, $M$ is not a fixed value over different iterations - it will increase rapidly as the iterations proceed because, at each iteration, as many as 26 neighbors of an active node will be inserted into the queue and only one node (the head) will be removed from it.

Hence $M$ can become very large, which makes sorting the queue very time-consuming. As the sorting takes place after each of the frequent node insertions, this becomes the bottleneck of the serial algorithm. In our experiments, we found that the value of $M$ depends upon the shape of the object: the fatter the object is, the more active nodes will accumulate in the queue from the previous iterations, so the larger the queue will become. For example, the cuboid model in Figure 5 is wide and this will cause a large number of voxels to be accumulated in the queue; as a result, for this model, the serial algorithm is 500 times slower than even the brute-force parallel algorithm, and thousands of times slower than the $i m_{-} p$ algorithm. This also explains why the serial algorithm took 13 seconds for the vessel model but an hour for the toy model, even though both models have the same $256^{3}$ resolution. This is because the values of $K$ and $M$ are much larger for the toy model than for the vessel model, even though the resolution $N$ of both models is the same.

The computational complexity of the $b f_{-} p$ algorithm is $O(N \times I)$, where $I$ is the number of iterations, which is a bounded value because after information is propagated to reach all interior nodes the iteration procedure will stop. At each iteration, all $N$ nodes of the dataset need to be evaluated regardless of whether they are active or not, however this is performed in a parallel mode by launching $N$ concurrent CUDA threads. As a result it could still be hundreds of times faster than the serial algorithm.

The complexity of $i m_{-} p$ is $O($ currentLength $\times I)$. It again performs in a parallel mode, but instead of evaluating all $N$ nodes at each iteration, it evaluates only currentLength nodes by launching currentLength concurrent CUDA threads. Here, currentLength is the number of the currently active nodes (recorded in currentArray) at each iteration - this can be thought of as equivalent to the length of the 


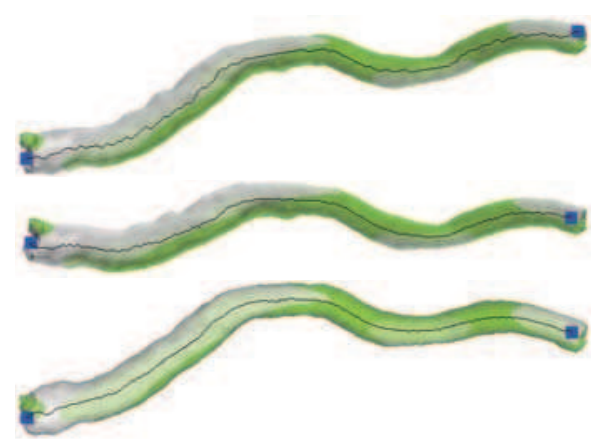

serial

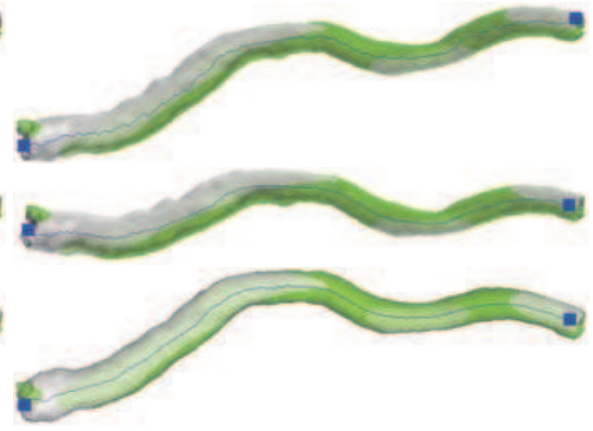

bf

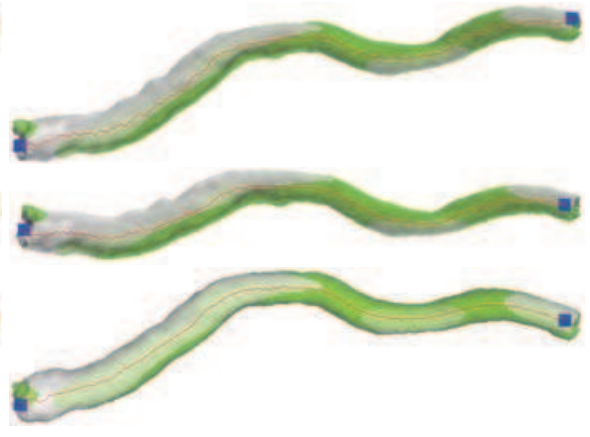

im

Fig. 4. Results produced by the three algorithms (left to right: serial, $b f_{-} p$ and $i m_{-} p$ ) for a blood vessel model (femoral artery) at three different data resolutions (top to bottom rows: $128^{3}, 256^{3}$ and $512^{3}$, respectively). In each row, the three algorithms produce identical results; in each column, the dataset with the higher resolution produces a smoother result. Please zoom in to see the details. The corresponding performance statistics for these images are shown in Table 1.

priority queue (the active set) in the serial algorithm. But the difference here is that only the currently new "active" nodes at the current iteration need to be inserted into nextArray. Previously active nodes in currentArray will be inserted into nextArray only if they are again active (that is, their weight is again changed) at the current iteration. As a result, the active set remains much smaller during the ping-pong process than in serial.

Intuitively, the size of the active set in $i m_{-} p$ (i.e., currentLength) at a certain iteration is the size (or area) of the wavefront patch shown in Fig. 3, which is a relatively smooth one-voxel-thick surface patch that propagates from the source point and is 'cut' by the object's surface. At each iteration, one wavefront is replaced by another onevoxel-thick wavefront in the active set. If $I$ is the number of such wavefront patches during the propagation, then typically $I$ is roughly of the same order as the diameter of the object i.e., $\sqrt[3]{N}$ for a volume of $N$ voxels).

In the serial algorithm, the size of the active set, $M$, is more like a sub-volume or volumetric fraction of the object - it is much thicker than our wavefront patch. This is caused by node accumulation - at each iteration, only one node is removed from the queue but up to 26 nodes are inserted into it. As a result, many historically active nodes are accumulated in the queue as the iterations progress, and the size of the active set can become as large as a volumetric fraction of the object, i.e., of the same order as $K$. This explains why it performs much more slowly than $i m_{-} p$

In theory, the $i m_{-} p$ algorithm could also be implemented on a multicore CPU, but the latest CPUs still have only very few cores (up to 8 cores, generally). As, typically, thousands of nodes have to be evaluated concurrently at each iteration, 8 cores are far too few to provide a suitable level of parallelism compared to what is available on a modern GPU.

\subsection{Comparison with latest curve-skeleton algorithms}

Curve skeletons are different from centerlines, as we outlined in Section 1, and are not as suitable as centerlines for direct use as flythrough paths due to their branches and their inability to pass through the predefined end points selected by the users. Nevertheless, for a greater insight into the performance of our method, we next compare the speed of our GPU centerline extraction with several state-of-the-art curve-skeleton algorithms.

Arcelli et al. [3] reported average times of 35 seconds for extracting the curve skeleton of $128^{3}$ resolution volumetric models, including similar dino and horse models to those in Table 1, on a $3 \mathrm{GHz}$ CPU. This implies that our method is hundreds of times faster (even more so since we used a higher resolution of $256^{3}$ for these two models).

We have run the binary code provided by the authors of [27] on the machine used for Table 1, and the curve-skeleton extraction time of their algorithm for the horse dataset is 19.52 seconds, which is slower than our $i m_{-} p$ method by a factor of almost 100 .

We also ran the binary code provided by the authors of [4], which is the most well known geometric approach for extracting curve skeletons directly from mesh surfaces. The curve-skeleton extraction time for the same horse model was 960 seconds, which is much slower than our methods and the other voxel-based methods. However, this method has a much lower memory consumption, since it does not need to store the volumetric datasets.

The method of Jalba et al. [20] uses GPU acceleration and requires, on a GTX 280 card, between 3 and 9 seconds (with an average of 4 seconds) to extract curve skeletons from models similar to the ones in Fig. 6, e.g. 5.3 seconds for horse and 3.8 seconds for hand. This compares to our 0.23 and 0.24 seconds, respectively, for the same models. Allowing for the fact that the GTX 690 GPU is 3 times faster than the GTX 280 GPU, our method remains 5 to 7 times faster, and it also has the advantage of a much simpler implementation.

As a final remark, we note that for applications such as fly-through path planning, which requires a single branchless centerline that passes through two user-defined end points, our algorithm is much more economical than these curve-skeleton methods. Even if such methods were to process the skeleton as efficiently as $i m_{-} p$, time-consuming postprocessing would still have to be performed to remove undesired minor branches from the skeleton to produce a major trunk as the final fly-through path.

\subsection{GPU Memory consumption}

The main memory consumption of the $i m_{-} p$ algorithm lies in three 3D arrays. Besides the $3 \mathrm{D}$ texture of $D F B_{\text {cost }}$ in float format, we also need a float 3D array weight and a Boolean 3D array mark in byte format. So for a $512^{3}$ dataset, e.g. the vessel and aorta in Table 1, the GPU memory consumption is $512^{3} \times(4+4+1)$ bytes, i.e., $128 \times 9 \mathrm{MB}=$ $1152 \mathrm{MB}$.

For larger datasets, such as $1024^{3}$, the memory consumption could become a problem for a single GPU. To alleviate this, one could use half-precision floating-point format, which occupies 16 bits. This is commonly employed in GPU computing and has produced acceptable results for many applications. The various Boolean arrays can be further compressed to use one bit per voxel instead of 1 byte per voxel. Furthermore, recent GPUs, such as the NVIDIA GeForce Titan, have 6 GB GPU memory, which is sufficient to handle a $1024^{3}$ dataset at halfprecision floating-point format. As such, we consider that our implementation is suitable for use with normal real-world volume datasets without further modification. In the future, we will investigate using multi-GPUs to deal with even larger datasets.

\subsection{Applications to virtual endoscopic simulation}

Our im_p centerline extraction algorithm can be used for compact shape description, segment-length measurement, automatic naviga- 

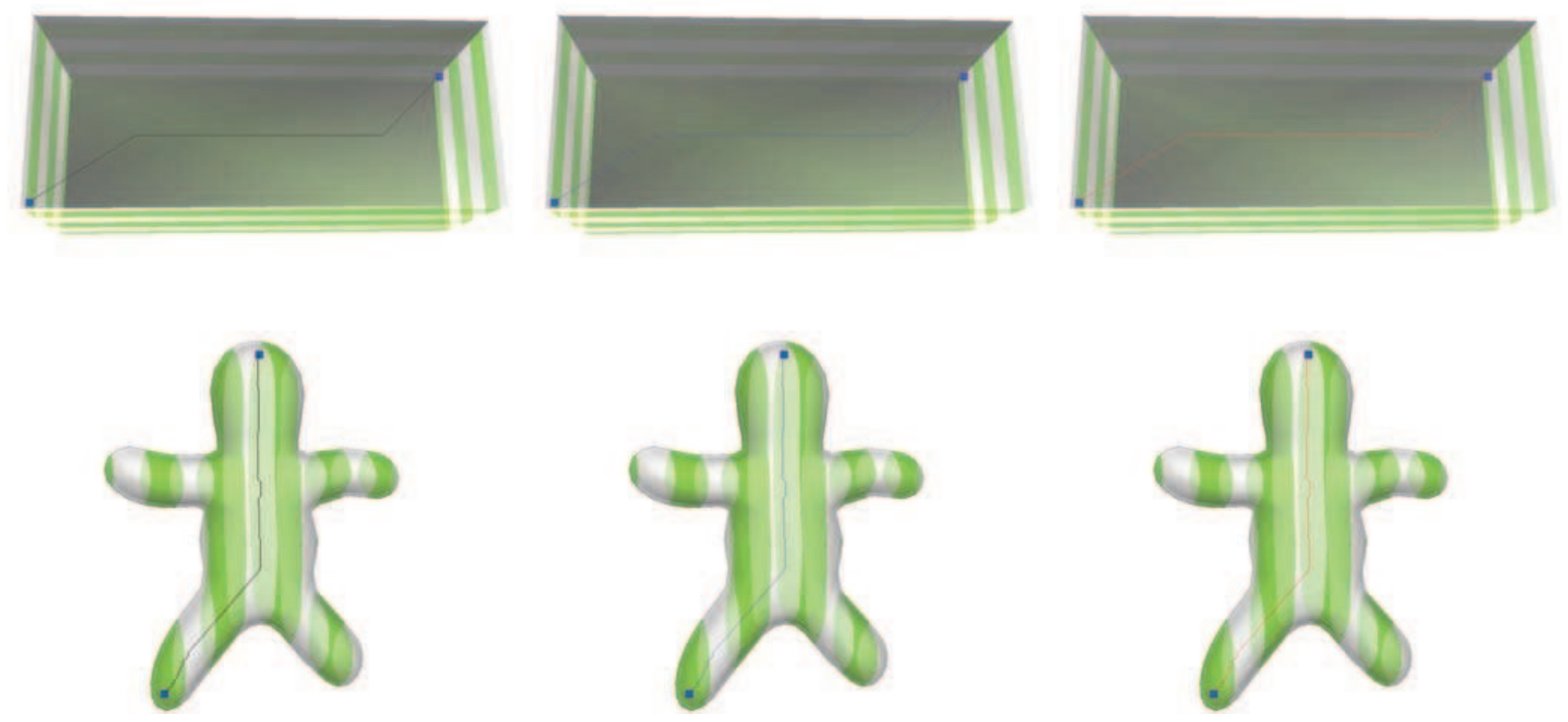

Fig. 5. More identical results produced by the three algorithms (from left to right: serial, $b f_{-} p$ and $i m_{-} p$ ) for the cuboid and doll models, respectively. Please zoom in to see the details. The corresponding performance statistics for these images are shown in Table 1.

tion, etc., in virtual endoscopic simulation applications.

It can provide flexible real-time navigation paths based on userspecified end points within a 3D virtual model acquired from a continuous sequence of 2D medical image slices scanned from the human colon, aiming to detect early-stage colon polyps. It can extract a centerline hundreds of times faster than the previous serial approach, in fact, so fast that the user will hardly notice any latency - in considerably less than a second in all of our experiments. This can be very helpful in assisting an interactive virtual endoscopic diagnostic process, as we show in the accompanying video, which uses the extracted centerline directly as the camera path (for the aneurism 2 dataset). The video shows that the camera path is highly centered and smooth, and never collides with the object wall.

The extracted centerlines can also provide accurate measurements of the locations of abnormalities. For example, once a polyp is detected during a colon navigation, the physician immediately knows its location and the distances to the two end points $S$ and $E$, which is useful for registration in surgery.

\section{Conclusion}

We have introduced a parallel algorithm, suited to modern GPU hardware, to extract centerlines automatically at interactive speed. Experiments have shown that the proposed algorithm is hundreds of times faster than the previous serial algorithm.

The proposed new algorithm im_ $p$ is not only fast, but also has other desirable features, including precision, connectivity, simplicity, and computational efficiency.

The algorithm can produce a 26-connected highly centered singular path which is exactly 1-voxel thick, by construction. The centeredness of our centerline is derived from the DFB field. No ad hoc adjustments are needed to push the path back to the center.

Since our centerlines are extracted from the MST in the DFB field, they inherit the simplicity of the paths in a tree structure. In other words, the algorithm generates centerlines of 1-voxel width between the two end points ( $S$ and $E$ ), with no 2-D manifolds or 3-D selfintersections. This is important for automatic camera-path planning, as it avoids folding or self-intersection of the navigation path thus removing the possibility of "Y" junctions that can cause ambiguity and hesitation in the traversal.
The centerlines produced have the correct tendency to stay close to the local center of the object, due to the fact that the computation is based on an accurate DFB field with the exact Euclidean values. Another advantage is that the centerlines produced can be smoother than those of the serial algorithm due to the low-pass filtering effect of the new algorithm.

The algorithm is robust and deterministic, and is easy to understand, implement and maintain as its main concept is straightforward due to the precise and concise centerline definition.

\section{REFERENCES}

[1] L. Antiga. Patient-Specific Modeling of Geometry and Blood Flow in Large Arteries. PhD thesis, Politecnico di Milano, Dipartimento di Bioingegneria, Italy, 2002.

[2] L. Antiga. VMTK - the vascular modeling toolkit. http://www.vmtk.org/index.html, 2012.

[3] C. Arcelli, G. Sanniti di Baja, and L. Serino. Distance-driven skeletonization in voxel images. IEEE TPAMI, 33(4):709-720, 2011.

[4] O. K.-C. Au, C.-L. Tai, H.-K. Chu, D. Cohen-Or, and T.-Y. Lee. Skeleton extraction by mesh contraction. ACM TOG, 27(3):44:1-44:10, Aug. 2008.

[5] S. Aylward and E. Bullitt. Initialization, noise, singularities, and scale in height ridge traversal for tubular object centerline extraction. IEEE Transactions on Medical Imaging, 21(2):61-75, Feb. 2002.

[6] X. Bai, L. Latecki, and W. yu Liu. Skeleton pruning by contour partitioning with discrete curve evolution. IEEE TPAMI, 29(3):449-462, 2007.

[7] I. Bitter, A. Kaufman, and M. Sato. Penalized-distance volumetric skeleton algorithm. IEEE TPAMI, 7(3):195-206, 2001.

[8] I. Bitter, M. Sato, M. Bender, K. McDonnell, A. Kaufman, and M. Wan. CEASAR: a smooth, accurate and robust centerline extraction algorithm. In Proc. IEEE Visualization, pages 45-52, 2000.

[9] A. Bleiweiss. GPU Accelerated Pathfinding. In Proceedings of the 23rd ACM SIGGRAPH/EG Symp. on Graphics Hardware, pages 65-74, Sarajevo, Bosnia-Herzegovina, 2008.

[10] H. Blum. A transformation for extracting new parameter of shape. In Models for the Perception of Speech and Visual Form, MA: MIT Press, 1967.

[11] T.-T. Cao, K. Tang, A. Mohamed, and T.-S. Tan. Parallel banding algorithm to compute exact distance transform with the GPU. In Proc. ACM 
SIGGRAPH Symp. on Interactive 3D Graphics and Games, pages 83-90, 2010.

[12] N. Cornea, D. Silver, and P. Min. Curve-skeleton properties, applications, and algorithms. IEEE TVCG, 13(3):530-548, May-June 2007.

[13] L. Costa and R. Cesar. Shape analysis and classification: Theory and practice, second edition. CRC Press, 2000.

[14] T. K. Dey and J. Sun. Defining and computing curve-skeletons with medial geodesic function. In Proc. SGP, pages 143-152. Eurographics, 2006.

[15] Y. Ge, D. R. Stelts, and D. J. Vining. 3D skeleton for virtual colonoscopy. In Proc. Intl. Conf. on Visualization in Biomedical Computing, pages 449-454, London, UK, UK, 1996. Springer-Verlag.

[16] M. S. Hassouna and A. A. Farag. Robust centerline extraction framework using level sets. In Proc. IEEE CVPR, pages 458-465, 2005.

[17] T. He and A. Kaufman. Collision detection for volumetric objects. In Proc. IEEE Visualization, pages 27-34, 1997.

[18] W. H. Hesselink and J. B. T. M. Roerdink. Euclidean skeletons of digital image and volume data in linear time by the integer medial axis transform. IEEE TPAMI, 30(12):2204-2217, 2008.

[19] H. Homann. Implementation of a 3D thinning algorithm. http://hdl.handle.net/1926/1292, Oct. 2007.

[20] A. C. Jalba, J. Kustra, and A. C. Telea. Surface and curve skeletonization of large 3D models on the GPU. IEEE TPAMI, 35(6):1495-1508, 2013.

[21] J. Latombe. Robot Motion Planning. Kluwer, second edition, 1991.

[22] J. Ma, S. Bae, and S. Choi. 3D medial axis point approximation using nearest neighbors and the normal field. The Visual Computer, 28(1):7$19,2012$.

[23] A. Meijster, J. B. T. M. Roerdink, and W. H. Hesselink. A general algorithm for computing distance transforms in linear time. In J. Goutsias, L. Vincent, and D. S. Bloomberg, editors, Mathematical Morphology and its Applications to Image and Signal Processing, pages 331-340. Kluwer, 2000.

[24] K. Palagyi and A. Kuba. Directional 3D thinning using 8 subiterations. In G. Bertrand, M. Couprie, and L. Perroton, editors, Discrete Geometry for Computer Imagery, volume 1568 of LNCS, pages 325-336. Springer, 1999.

[25] G. Peyre and L. Cohen. Geodesic computations for fast and accurate surface remeshing and parameterization. In Progress in Nonlinear Differential Equations and Their Applications, volume 63, pages 151-171. Springer LNCS, 2005. www . ceremade. dauphine. fr/ peyre.

[26] C. Pudney. Distance-ordered homotopic thinning: A skeletonization algorithm for 3D digital images. CVIU, 72(3):404-413, 1998.

[27] D. Reniers, J. van Wijk, and A. Telea. Computing multiscale curve and surface skeletons of genus 0 shapes using a global importance measure. IEEE TVCG, 14(2):355-368, 2008.

[28] R. J. Sadleir and P. F. Whelan. Fast colon centreline calculation using optimised 3D topological thinning. Computerized Medical Imaging and Graphics, 29(4):251-258, 2005.

[29] T. Saito and J.-I. Toriwaki. New algorithms for Euclidean distance transformation of an $n$-dimensional digitized picture with applications. Pattern Recognition, 27(11):1551-1565, 1994.

[30] M. Schaap, C. Metz, T. van Walsum, A. van der Giessen, A. Weustinck, N. Mollet, C. Bauer, H. Bogunovic, C. Castro, X. Deng, E. Dikici, T. O’Donnell, M. Frenay, O. Friman, M. Hoyos, P. Kitslaar, A. Szymczak, H. Tek, C. Wang, S. Warfield, S. Zambal, Y. Zhang, G. Kresting, and W. Niessen. Standardized evaluation methodology and reference database for evaluating coronary artery centerline extraction algorithms. Med Image Anal, 13(5):701-714, 2009.

[31] J. Schneider, M. Kraus, and R. Westermann. GPU-based euclidean distance transforms and their application to volume rendering. In A. Ranchordas, J. Pereira, H. Aranjo, and J. Tavares, editors, Computer Vision, Imaging and Computer Graphics. Theory and Applications, volume 68 of Communications in Computer and Information Science, pages 215-228. Springer-Verlag, 2010.

[32] J. Sethian. Level Set Methods and Fast Marching Methods, second edition. Cambridge Univ. Press, 2002.

[33] E. Smistad. GPU-Based Airway Tree Segmentation and Centerline Extraction for Image Guided Bronchoscopy. PhD thesis, Norwegian University of Science and Technology, 2012.

[34] E. Smistad, A. Elster, and F. Lindseth. Gpu accelerated segmentation and centerline extraction of tubular structures from medical images. Intl. J. Computer Assisted Radiology and Surgery, pages 1-15, 2013.

[35] E. Smistad, A. C. Elster, and F. Lindseth. GPU-based airway tree seg- mentation and centerline extraction for image guided bronchoscopy. In Proc. Norsk Informatik Konferanse. Akademika Forlag, 2012.

[36] A. Sobiecki, H. Yasan, A. Jalba, and A. Telea. Qualitative comparison of contraction-based curve skeletonization methods. In C. Hendriks, G. Borgefors, and R. Strand, editors, Mathematical Morphology and Its Applications to Signal and Image Processing, volume 7883 of LNCS, pages 425-439. Springer-Verlag, 2013.

[37] A. Tagliasacchi, I. Alhashim, M. Olson, and H. Zhang. Mean curvature skeletons. CGF, 31(5):1735-1744, 2012.

[38] R. Van Uitert and R. Summers. Automatic correction of level set based subvoxel precise centerlines for virtual colonoscopy using the colon outer wall. IEEE Trans. Med. Imag., 26(8):1069-1078, 2007.

[39] M. Wan, F. Dachille, and A. Kaufman. Distance-field based skeletons for virtual navigation. In Proc. IEEE Visualization, pages 239-560, 2001.

[40] M. Wan, Z. Liang, Q. Ke, L. Hong, I. Bitter, and A. Kaufman. Automatic centerline extraction for virtual colonoscopy. IEEE Trans. Med. Imag., 21(12):1450-1460, Dec. 2002.

[41] Y. Zhou and A. Toga. Efficient skeletonization of volumetric objects. IEEE TVCG, 5(3):196-209, 1999. 

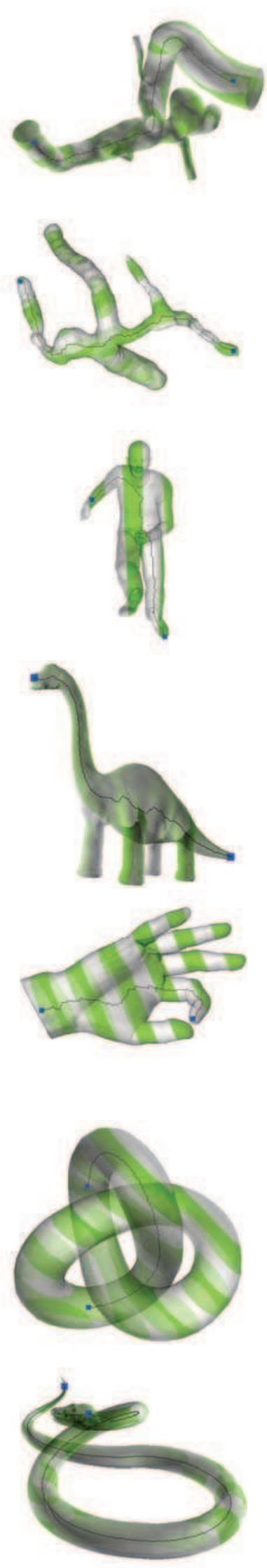
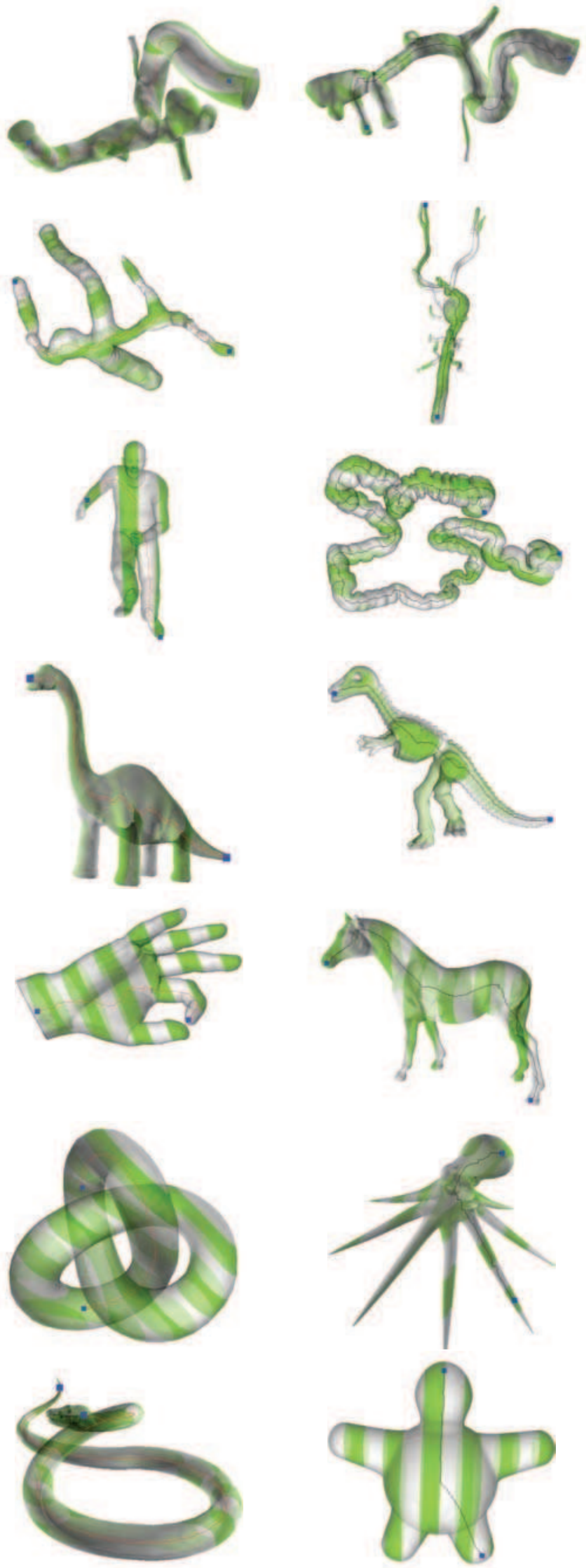
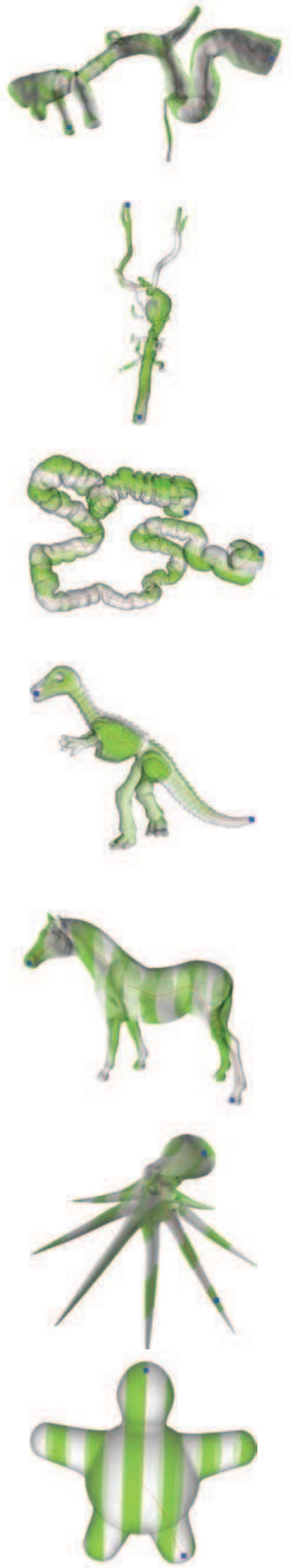

Fig. 6. Non-identical results produced by serial (left) and $i m_{-} p$ (right) algorithms. Please zoom in to see the details. From left to right, top to bottom, the datasets are aneurism 1 , aneurism 2 , aneurism 3 , aorta, ben, colon, dino, dinoSkeleton, hand, horse, knot, octopus, snake, and toy, respectively. 